\title{
Mott physics and spin fluctuations: a unified framework
}

\author{
Thomas Ayral ${ }^{1,2, *}$ and Olivier Parcollet ${ }^{2}$ \\ ${ }^{1}$ Centre de Physique Théorique, Ecole Polytechnique, CNRS-UMR7644, 91128 Palaiseau, France \\ ${ }^{2}$ Institut de Physique Théorique (IPhT), CEA, CNRS, URA 2306, 91191 Gif-sur-Yvette, France
}

\begin{abstract}
We present a formalism for strongly correlated electrons systems which consists in a local approximation of the dynamical three-leg interaction vertex. This vertex is self-consistently computed with a quantum impurity model with dynamical interactions in the charge and spin channels, similar to dynamical mean field theory (DMFT) approaches. The electronic self-energy and the polarization are both frequency and momentum dependent. The method interpolates between the spinfluctuation or GW approximations at weak coupling and the atomic limit at strong coupling. We apply the formalism to the Hubbard model on a two-dimensional square lattice and show that as interactions are increased towards the Mott insulating state, the local vertex acquires a strong frequency dependence, driving the system to a Mott transition, while at low enough temperatures the momentum-dependence of the self-energy is enhanced due to large spin fluctuations. Upon doping, we find a Fermi arc in the one-particle spectral function, which is one signature of the pseudo-gap state.
\end{abstract}

PACS numbers:

Strongly-correlated electronic systems like hightemperature cuprate superconductors are a major challenge in condensed-matter physics.

One theoretical approach to cuprates emphasizes the effect of long-range bosonic fluctuations on the electronic fluid, for example long-range antiferromagnetic (AF) fluctuations due to a quantum critical point [16]. These bosonic fluctuations are also central to approaches such as the two-particle self-consistent approximation (TPSC [7-11]), the GW approximation [12] and the fluctuation-exchange approximation (FLEX [13]).

Another approach focusses, following Anderson [14], on describing the Mott transition and the doped Mott insulator. In recent years, dynamical mean field theory (DMFT) [15] and its cluster extensions like CDMFT $[16,17]$ or DCA [18-20] have allowed for tremendous theoretical progress on the Mott transition both for models and realistic computations of strongly correlated materials [21]. In particular, numerous works have been devoted to the one-band Hubbard model, mapping out its phase diagram, studying the $d$-wave superconducting order and the pseudogap [22-45]. Cluster DMFT is one of the few methods designed for the strong-interaction regime to have a simple control parameter, namely the size $N_{c}$ of the cluster or the momentum resolution of the electronic self-energy. It interpolates between the DMFT solution $\left(N_{c}=1\right)$ and the exact solution of the Hubbard model $\left(N_{c}=\infty\right)$. Despite its success, this method nonetheless suffers from severe limitations: $i$ ) it does not include the effect of long-range bosonic modes of wavelengths larger than the cluster size; ii) the negative sign problem of continuous-time quantum Monte Carlo has so far precluded the convergence of the cluster solutions with respect to $N_{c}$ in the most important regimes like the pseudogap; iii) the k-resolution of the self-energy is still quite coarse in DCA (typically 8 or 16 patches in the Brillouin zone, see e.g. [31, 33, 45, 46]), or relies on uncontrolled a posteriori "periodization" techniques in CDMFT [17].

Several directions beyond cluster DMFT methods are currently under investigation to address these issues, such as GW + DMFT [47-53], the DГA method [54-57], the dual fermion [58] and dual boson methods [59, 60], or combinations of DMFT with functional renormalization group methods [61].

In this letter, we discuss a simple formalism that unifies the two points of view mentioned above. It is designed to encompass both Mott physics à la DMFT and the effect of medium and long-range bosonic modes. It interpolates between the atomic limit in the stronginteraction regime and the "fluctuation-exchange" limit in the weak-interaction regime. It consists in decoupling the electron-electron interaction term by HubbardStratonovich bosonic fields and making a local selfconsistent approximation of the lattice's electron-boson one-particle irreducible vertex, using a quantum impurity model similar to the one used in DMFT. It can be formally derived from a functional of the vertex given by three-particle irreducible diagrams [62, 63]. In the following, we will therefore denote this method as a triplyirreducible local expansion, or TRILEX. Already at the single-site level, it produces, in some parameter regimes, a momentum-dependent self-energy and polarization, at a small computational cost, similar to solving Extended DMFT (EDMFT) [64-66]. In the following, we first introduce the method; we then present the solution of the single-site version of TRILEX for the two-dimensional Hubbard model.

We focus on the Hubbard model defined by the following Hamiltonian:

$$
H=\sum_{i j} t_{i j} c_{i}^{\dagger} c_{j}+U \sum_{i} n_{i \uparrow} n_{i \downarrow}
$$

The indices $i, j$ denote lattice sites, $\sigma=\uparrow, \downarrow, c_{i \sigma}^{\dagger}$ and $c_{i \sigma}$ 


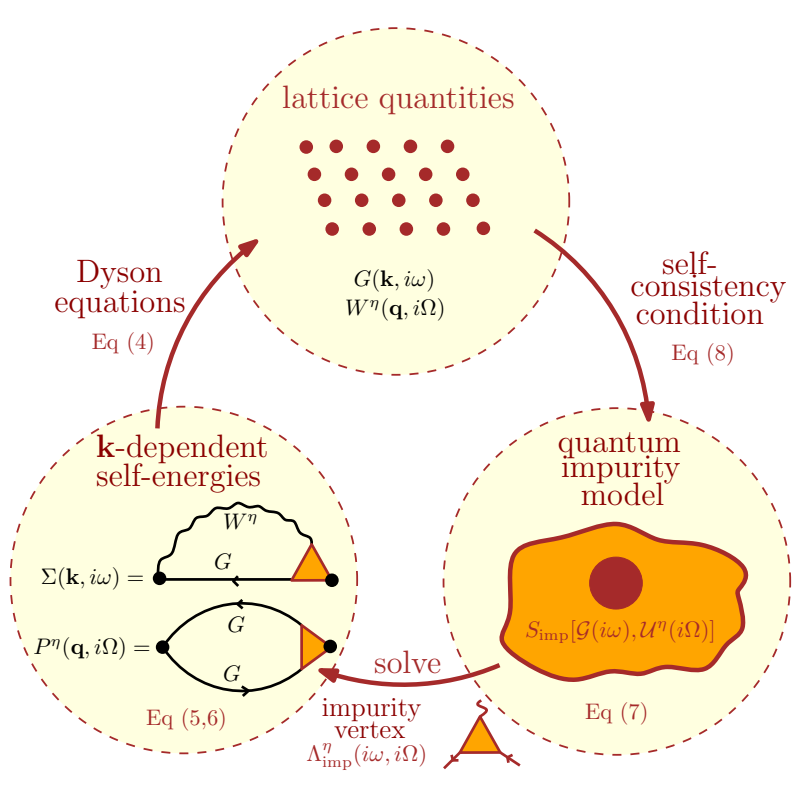

Figure 1: (color online) Description of the TRILEX method

are fermionic creation and annihilation operators, and $n_{i \sigma} \equiv c_{i \sigma}^{\dagger} c_{i \sigma} . \quad t_{i j}$ is the tight-binding hopping matrix ( $t_{i j}=t\left(t^{\prime}\right)$ for (next-)nearest-neighbors), while $U$ is the on-site Coulomb interaction. We rewrite the operators of the interaction term as:

$$
U n_{i \uparrow} n_{i \downarrow}=\frac{1}{2} \sum_{I} U^{I} n_{i}^{I} n_{i}^{I}
$$

where $U^{I}$ is the bare interaction in channel $I$, and $n_{i}^{I} \equiv \sum_{\sigma \sigma^{\prime}} c_{i \sigma}^{*} \sigma_{\sigma \sigma^{\prime}}^{I} c_{i \sigma^{\prime}}$ where $\sigma^{0}=\mathbf{1}$ and $\sigma^{x, y, z}$ are the Pauli matrices. In this paper, we consider two decouplings: (a) in the charge and vector spin channel $(I=0, x, y, z)$ (" $x y z$-decoupling"), $U^{x}=U^{y}=U^{z} \equiv U^{\mathrm{sp}}$ and $U^{0} \equiv U^{\mathrm{ch}}, U^{\mathrm{sp}}$ and $U^{\mathrm{ch}}$ satisfy: $U=U^{\mathrm{ch}}-3 U^{\mathrm{sp}}$; (b) in the charge and longitudinal spin channel only $(I=0, z)$ (" $z$-decoupling"), $U=U^{\text {ch }}-U^{\text {sp }}$. In both cases, we have two channels, denoted as $\eta=\mathrm{ch}$, sp. In this paper, we fix the ratio to $U^{\text {ch }}=U / 2$ and $U^{\text {sp }}=-U / 6$ ( $x y z$ decoupling) and $U^{\text {ch }}=U / 2$ and $U^{\text {sp }}=-U / 2(z$ decoupling). We now decouple (2) using real bosonic Hubbard-Stratonovich fields $\phi_{i}^{I}(\tau)$ in each channel and at each lattice site, so that the action becomes:

$$
\begin{aligned}
S_{\text {latt }}= & \int_{0}^{\beta} d \tau \sum_{i j} c_{i \sigma \tau}^{*}\left\{\partial_{\tau}+t_{i j}\right\} c_{j \sigma \tau} \\
& +\sum_{i, I}\left[\frac{1}{2}\left(U^{I}\right)^{-1} \phi_{i \tau}^{I} \phi_{i \tau}^{I}+\lambda^{I} \phi_{i \tau}^{I} n_{i \tau}^{I}\right]
\end{aligned}
$$

$c_{i \sigma \tau}^{*}$ and $c_{i \sigma \tau}$ are conjugate $\beta$-antiperiodic Grassmann fields, and $\lambda^{I}=1$. We are now dealing with an interacting lattice problem with a local electron-boson coupling. The lattice Green's functions $G(\mathbf{k}, i \omega)$ and $W^{\eta}(\mathbf{q}, i \Omega)$ (the Fourier transforms of $-\left\langle c_{i \sigma \tau} c_{j \sigma 0}^{*}\right\rangle$ and $-\left\langle\phi_{i \sigma \tau}^{\eta} \phi_{j \sigma 0}^{\eta}\right\rangle$, respectively) are given by Dyson equations:

$$
\begin{aligned}
G(\mathbf{k}, i \omega) & =[i \omega+\mu-\epsilon(\mathbf{k})-\Sigma(\mathbf{k}, i \omega)]^{-1} \\
W^{\eta}(\mathbf{q}, i \Omega) & =U^{\eta}\left[1-U^{\eta} P^{\eta}(\mathbf{q}, i \Omega)\right]^{-1}
\end{aligned}
$$

$\mathbf{k}$ and $\mathbf{q}$ are momentum variables, $i \omega(i \Omega)$ stands for a fermionic (bosonic) Matsubara frequency, $\epsilon(\mathbf{k})$ is the Fourier transform of $t_{i j}$, and $\mu$ is the chemical potential. The fermionic and bosonic self-energies $\Sigma$ and $P^{\eta}$ are given by the exact expressions (written here for the paramagnetic normal phase) (see e.g [67]):

$$
\begin{aligned}
& \Sigma(\mathbf{k}, i \omega)=-\sum_{\substack{\mathbf{q}, i \Omega, \eta=\mathrm{ch}, \mathrm{sp}}} m_{\eta} \lambda^{\eta} G_{i \omega+i \Omega}^{\mathbf{q}+\mathbf{k},} W_{\mathbf{q}, i \Omega}^{\eta} \Lambda_{\mathbf{k}, \mathbf{q},}^{\eta}, \\
& P^{\eta}(\mathbf{q}, i \Omega)=2 \sum_{\mathbf{k}, i \omega} \lambda^{\eta} G_{i \omega+i \Omega}^{\mathbf{q}+\mathbf{k},}, G_{\mathbf{k}, i \omega} \Lambda_{\mathbf{k}, \mathbf{q},}^{\eta}
\end{aligned}
$$

Here, $m_{\mathrm{ch}}=1, m_{\mathrm{sp}}=3$ (xyz decoupling) or $m_{\mathrm{sp}}=1$ ( $z$ decoupling $). \Lambda^{\eta}(\mathbf{q}, \mathbf{k}, i \omega, i \Omega)$ is the exact one-particle irreducible electron-boson coupling (or Hedin) vertex, namely the effective interaction between electrons and bosons renormalized by electronic interactions.

The main point of this paper consists in approximating the vertex $\Lambda^{\eta}(\mathbf{q}, \mathbf{k}, i \omega, i \Omega)$ by the local, but twofrequency-dependent $\Lambda_{\text {imp }}^{\eta}(i \omega, i \Omega)$ computed from a selfconsistent quantum impurity problem:

$$
\Lambda^{\eta}(\mathbf{q}, \mathbf{k}, i \omega, i \Omega) \approx \Lambda_{\mathrm{imp}}^{\eta}(i \omega, i \Omega)
$$

This strategy radically differs from DMFT, EDMFT and GW + DMFT which approximate the self-energy $\Sigma$ (and $P$ ), not $\Lambda$. It implies that our $\Sigma$ and $P$ (computed from $(5 \mathrm{a}-5 \mathrm{~b}))$ are, in some parameter regimes, strongly momentum-dependent while containing local vertex corrections which will be essential to capture Mott physics (see also [50]). Formally, DMFT is a local approximation of the two-particle irreducible Luttinger-Ward functional $\Phi_{\text {LW }}[G][15,68]$. In contrast, our approximation can be defined as a local approximation of the three-particle irreducible functional $\mathcal{K}[G, W, \Lambda]$ introduced in $[62,63]$ as a generalization of $\Phi_{\mathrm{LW}}$ to higher degrees of irreducibility. We therefore denote it as TRILEX, triply-irreducible local expansion. It makes it exact in the limit of infinite dimensions. The formal derivation of the method will be provided elsewhere [69].

The action of the impurity model reads:

$$
\begin{aligned}
S_{\mathrm{imp}}= & -\iint_{0}^{\beta} d \tau d \tau^{\prime} \sum_{\sigma} c_{\sigma \tau}^{*} \mathcal{G}\left(\tau-\tau^{\prime}\right) c_{\sigma \tau^{\prime}} \\
& +\frac{1}{2} \sum_{I} \iint_{0}^{\beta} d \tau d \tau^{\prime} n_{\tau}^{I} \mathcal{U}^{I}\left(\tau-\tau^{\prime}\right) n_{\tau^{\prime}}^{I}
\end{aligned}
$$

This is an Anderson quantum impurity with retarded charge-charge $(I=0)$ and spin-spin $(I=x, y, z$ in 


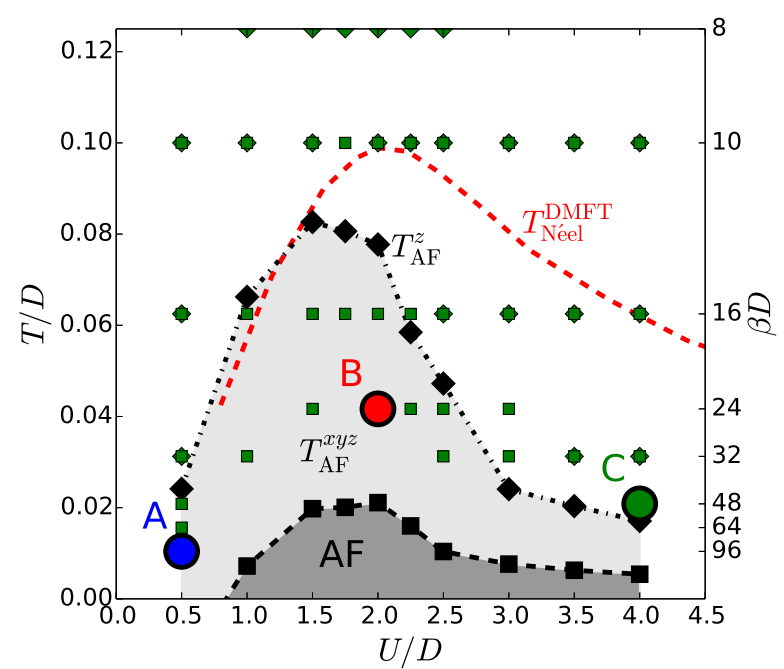

Figure 2: (color online) ( $T, U)$ Phase diagram (half-filling, $\left.t^{\prime}=0\right)$. The black diamonds (resp. squares) denote $T_{\mathrm{AF}}^{z}$ (resp. $T_{\mathrm{AF}}^{x y z}$ ); the dashed lines are guides to the eye. The green diamonds (resp. squares) denote converged TRILEX solutions in the $z$ (resp. $x, y, z)$ decoupling. $\mathrm{A}, \mathrm{B}$, and $\mathrm{C}$ are defined as $\mathrm{A}: \beta D=96, U / D=0.5, \mathrm{~B}: \beta D=24, U / D=2$, C: $\beta D=48, U / D=4$. The red dotted line denotes $T_{\text {Néel }}^{D M F T}$ for the square lattice (from [70]).

the $x y z$-decoupling, $I=z$ in the $z$-decoupling) interactions. The bosonic fields $\phi^{I}$ have been integrated out to obtain a fermionic action with retarded interactions amenable to numerical computations. We compute the fermionic three-point correlation functions to reconstruct the electron-boson vertex $\Lambda_{\text {imp }}$ (as shown in the Suppl. Mat., section B). Finally, $\mathcal{G}$ and $\mathcal{U}^{\eta}$ derive from the selfconsistency conditions as follows:

$$
\begin{aligned}
\mathcal{G}^{-1}(i \omega) & =G_{\mathrm{loc}}^{-1}(i \omega)+\Sigma_{\mathrm{loc}}(i \omega) \\
{\left[\mathcal{U}^{\eta}\right]^{-1}(i \omega) } & =\left[W_{\mathrm{loc}}^{\eta}\right]^{-1}(i \omega)+P_{\mathrm{loc}}^{\eta}(i \omega)
\end{aligned}
$$

where, for any $X, X_{\text {loc }}(i \omega) \equiv \sum_{\mathbf{k}} X(\mathbf{k}, i \omega)$. At convergence, this ensures that $G_{\mathrm{loc}}=G_{\mathrm{imp}}$ and $W_{\mathrm{loc}}^{\eta}=W_{\mathrm{imp}}^{\eta}$. $W^{\eta}$ and the susceptibility $\chi^{\eta}$ are related by:

$$
W^{\eta}(\mathbf{q}, i \Omega)=U^{\eta}-U^{\eta} \chi^{\eta}(\mathbf{q}, i \Omega) U^{\eta}
$$

The computational scheme is illustrated in Fig. 1. From the impurity electron-boson vertex $\Lambda_{\text {imp }}$, we compute $\Sigma(\mathbf{k}, i \omega)$ and $P^{\eta}(\mathbf{q}, i \Omega)$, which are then used to compute $\mathcal{G}$ and $\mathcal{U}^{\eta}$ for (7). We solve the quantum impurity model exactly by a continuous-time quantum MonteCarlo algorithm [71] in the hybridization expansion [72] with retarded density-density [73] and vector spin-spin interactions [74]. The computation of the three-point functions are implemented as described in [75]. We iterate until convergence is reached. Our implementation is based on the TRIQS library [76].

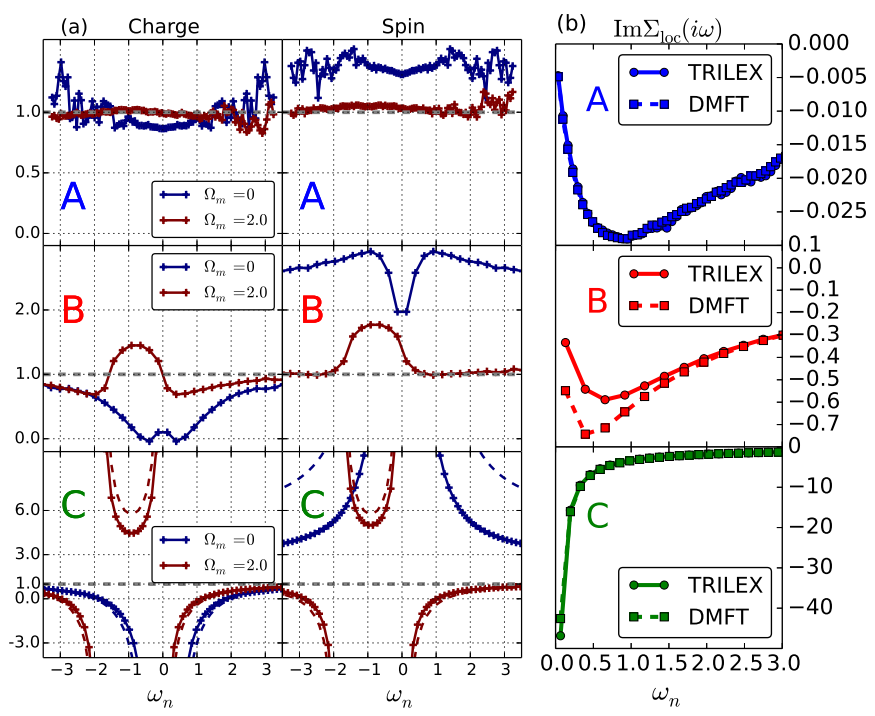

Figure 3: (color online) Left: Evolution of the local vertex $\operatorname{Re} \Lambda^{\eta}\left(i \omega_{n}, i \Omega_{m}\right)$ (half-filling, $t^{\prime}=0$ ). A, B and C are defined in Fig. 2. The dashed lines denote the atomic vertex $\Lambda_{\mathrm{at}}^{\eta}$ (Eq. (A1)). Right: $\operatorname{Im} \Sigma_{\text {loc }}\left(i \omega_{n}\right)$ for TRILEX and DMFT (paramagnetic phase).

TRILEX provides a unified framework for spinfluctuation approaches and Mott physics. Indeed, (i) at small interaction strengths, the local vertex reduces to the bare, frequency-independent vertex $\lambda^{\eta}$ so that $\Sigma$ is given by one-loop self-consistent diagrams, as in spin fluctuation theory in its simplest form (spin channel only), the GW approximation (charge channel only), or in FLEX limited to particle-hole diagrams; similarly, $P^{\eta}$ becomes equal to the "bubble" diagram; (ii) it is exact in the atomic limit $(t=0)$ : the effective local action turns into an atomic problem, $\Lambda$ into the atomic vertex $\Lambda_{\text {at }}$ (Eq.A1), and $\Sigma$ and $P$ become local, atomic self-energies.

Let us now apply the TRILEX method to the Hubbard model on a square lattice. All energies are given in units of the half-bandwidth $D=4|t|$. The Brillouin zone is discretized on a $64 \times 64$ momentum mesh. We restrict ourselves to the paramagnetic normal phase.

In Fig. 2, we present the phase diagram in the $(T, U)$ plane at half-filling. We obtain converged solutions of the TRILEX scheme above a temperature denoted $T_{\mathrm{AF}}^{x y z}$ (resp. $T_{\mathrm{AF}}^{z}$ ) for the $x y z$-decoupling (resp. $z$-decoupling). The evolution of the local vertex and self-energy (resp. lattice self-energy and polarizations) is presented in Fig.3 (resp. Fig. 4) for the points A, B and C of Fig. 2, in the $x y z$-decoupling. At weak coupling (point A), the local vertex $\Lambda$ reduces to the bare vertex $\lambda=1$ at large frequencies, up to numerical noise (Fig. 3a, upper panels). The spin polarization (hence the spin susceptibility, see Eq. (9)) becomes sharply peaked at the AF wavevector $\mathbf{Q}=(\pi, \pi)$ (Fig. 4, upper panels), reflecting the nesting features of the Fermi surface. As a result, the self-energy 

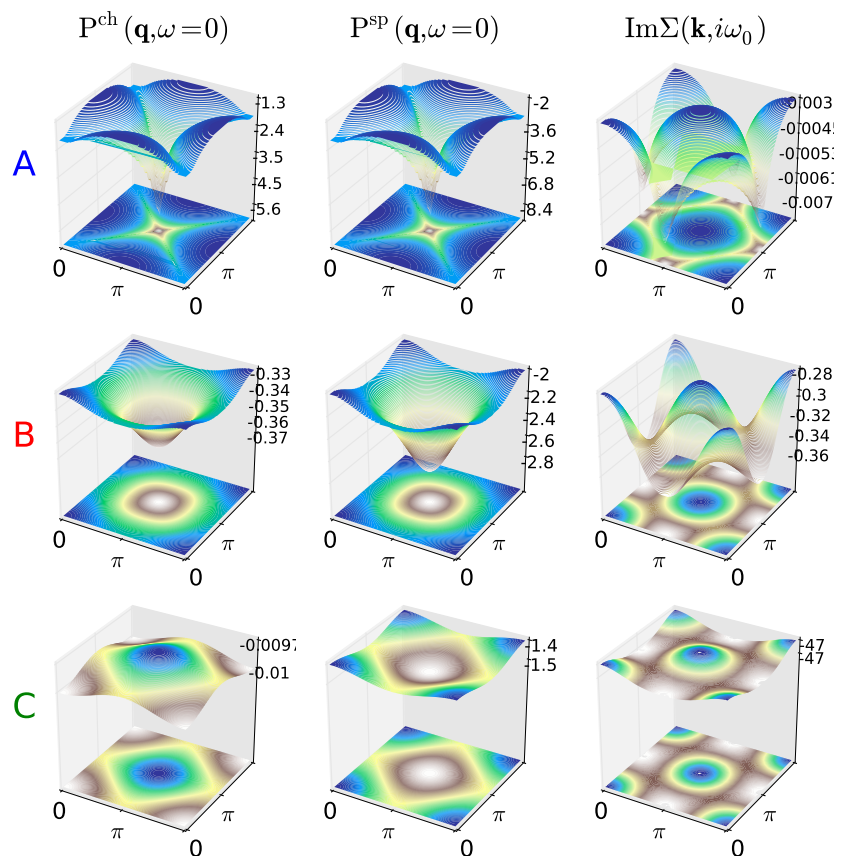

Figure 4: (color online) Momentum-dependence of the selfenergy and polarization (half-filling, $t^{\prime}=0$ ). $\mathrm{A}, \mathrm{B}$ and $\mathrm{C}$ are defined in Fig. 2. Left: $\operatorname{Re} P^{\text {ch }}(\mathbf{q}, \omega=0)$. Middle: $\operatorname{Re} P^{\mathrm{sp}}(\mathbf{q}, \omega=0)$. Right: $\operatorname{Im} \Sigma\left(\mathbf{k}, i \omega_{0}\right)$.

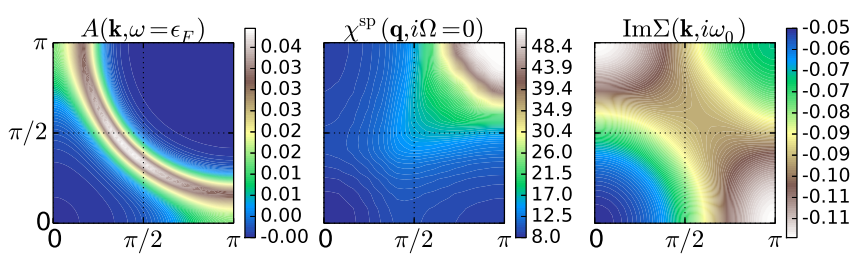

Figure 5: (color online) From left to right: $A(\mathbf{k}, \omega=0)$, $\chi(\mathbf{q}, i \Omega=0)$ and $\operatorname{Im} \Sigma\left(\mathbf{k}, i \omega_{0}\right)$ in the doped case: $U / D=1.8$, $t^{\prime}=-0.4 t, \beta D=96, \delta=10 \%$. (xyz-decoupling)

acquires a strong $\mathbf{k}$-dependence at $(\pi, 0)$ (Fig. 4$)$, but its local part is the same as the DMFT self-energy (Fig.3b). At strong coupling (point $\mathrm{C}$ ), the vertex becomes similar to the atomic vertex (Fig. 3a, lower panels). Furthermore, the self-energy and polarization are weakly momentum-dependent (Fig. 4, lower panels), in agreement with cluster DMFT calculations; the self-energy of TRILEX is very close to the DMFT self-energy (Fig. 3b). Finally, at intermediate coupling (point B), $\Lambda_{\text {imp }}$ acquires frequency structures which interpolate between $\mathrm{A}$ and $\mathrm{C}$ (Fig. 3a, middle panels), while $\Sigma$ is strongly momentumdependent and its local part departs from the DMFT self-energy (Fig. 3b, middle panels).

The temperature $T_{\mathrm{AF}}^{x y z / z}$ is determined by extrapolating the inverse static AF susceptibility (Fig.A.1 in Suppl. Mat.). It is reduced with respect to the Néel temperature computed in DMFT [70] as a result of nonlocal fluctuations beyond DMFT. Furthermore, $T_{\mathrm{AF}}^{x y z} \ll T_{\mathrm{AF}}^{z}$. As a consequence of the apparent divergence in the spin susceptibility at low temperatures (Fig. A.1), caused by a vanishing denominator of $W^{\eta}$ (Eq. 4b), we cannot obtain converged results in the close vicinity of and below $T_{\mathrm{AF}}$. Whether we have an actual AF transition or finite but very large correlation lengths (as seen e.g in [56]), could be decided by generalizing the present formalism to the symmetry-broken phase. Contrary to cluster DMFT, the susceptibilities are not by-products of the calculation, but directly enter the self-consistency loop through $W^{\eta}$ (see Eq.(9)). We thus cannot converge paramagnetic solutions below an AF phase transition.

Let us now turn to the effect of doping. In Fig. 5, we present results for $t^{\prime}=-0.4 t, \beta D=96$ and an intermediate interaction strength $(U=1.8$, close to point B). The spectral function displays Fermi arcs (Fig. 5, left panel), as observed in experiments [77] and in cluster DMFT [35, 37, 38, 42-44, 78]. Let us emphasize that this is obtained by solving a single-site quantum impurity problem, a far easier task than solving cluster impurities. The Fermi arc is a consequence of the large static spin susceptibility at the AF wavevector (Fig. 5, middle panel), which translates into a large imaginary part of the self-energy (Fig. 5, right panel). The corresponding variation of the spectral weight on the Fermi surface is rather mild due to the moderate correlation length ( $\xi_{\mathrm{AF}} \sim 2$ unit spacings) for these parameters.

Alternative self-consistency conditions are possible, e.g $\chi_{\mathrm{loc}}^{\eta}=\chi_{\mathrm{imp}}^{\eta}$ instead of $W_{\mathrm{loc}}^{\eta}=W_{\mathrm{imp}}^{\eta}$ would enforce sum rules on two-particle quantities that are key to preserving the Mermin-Wagner theorem in [7, 55]. However, this leads to a positive $\mathcal{U}^{\mathrm{sp}}(\tau)$ and hence to a severe sign problem in the quantum Monte-Carlo at low temperatures.

In conclusion, we have presented the TRILEX formalism, which encompasses long-range spin fluctuation effects and Mott physics in a unified way. Like DMFT, it can be systematically controlled by extending it to cluster schemes that interpolate between the single-site approximation studied in this paper, and the exact solution of the model. We expect that the convergence of the method as a function of the cluster size will strongly depend on the decoupling channel and, when done in the physically relevant channel, will be faster than cluster DMFT methods. Furthermore, because the competition between spin fluctuations and Mott physics can be described already at the single-site level, the method may be a good starting point for correlated multiorbital systems where spin fluctuations play an important role, like pnictides superconductors.

We acknowledge useful discussions S. Andergassen, S. Biermann, M. Ferrero, A. Georges, D. Manske, G. Misguich, J. Otsuki, A. Toschi. We thank H. Hafermann for help with implementing the measurement of the threepoint correlation function. This work is supported by the FP7/ERC, under Grant Agreement No. 278472MottMetals. 
* Electronic address: thomas.ayral@polytechnique.edu

[1] A. V. Chubukov, D. Pines, and J. Schmalian, in The Physics of Conventional and Unconventional Superconductors (2002) Chap. 22, p. 1349, arXiv:0201140 [condmat] .

[2] K. B. Efetov, H. Meier, and C. Pépin, Nature Physics 9, 442 (2013), arXiv:1210.3276 .

[3] Y. Wang and A. Chubukov, Physical Review B 90, 035149 (2014), arXiv:1401.0712 .

[4] M. A. Metlitski and S. Sachdev, Physical Review B 82, 075128 (2010).

[5] F. Onufrieva and P. Pfeuty, Physical Review Letters 102, 207003 (2009).

[6] F. Onufrieva and P. Pfeuty, Physical Review Letters 109, 257001 (2012).

[7] Y. Vilk, L. Chen, and A.-M. S. Tremblay, Physical Review B 49, 0 (1994).

[8] A.-M. Daré, Y. M. Vilk, and A.-M. S. Tremblay, Physical Review B 53, 14236 (1996).

[9] Y. M. Vilk and A.-M. S. Tremblay, EPL (Europhysics Letters) 33, 159 (1996).

[10] Y. Vilk and A.-M. S. Tremblay, Journal de Physique I, 1 (1997), arXiv:9702188v3 [arXiv:cond-mat] .

[11] A.-M. S. Tremblay, in Theoretical methods for Strongly Correlated Systems (2011) arXiv:arXiv:1107.1534v2 .

[12] L. Hedin, Physical Review 139, 796 (1965).

[13] N. Bickers and D. Scalapino, Annals of Physics 206251, 206 (1989).

[14] P. Anderson, Science 235, 1196 (1987).

[15] A. Georges, W. Krauth, and M. J. Rozenberg, Reviews of Modern Physics 68, 13 (1996).

[16] A. I. Lichtenstein and M. I. Katsnelson, Physical Review B 62, R9283 (2000).

[17] G. Kotliar, S. Savrasov, G. Pálsson, and G. Biroli, Physical Review Letters 87, 186401 (2001).

[18] M. H. Hettler, A. N. Tahvildar-Zadeh, M. Jarrell, T. Pruschke, and H. R. Krishnamurthy, Physical Review B 58, R7475 (1998), arXiv:9803295 [cond-mat] .

[19] M. H. Hettler, M. Mukherjee, M. Jarrell, and H. R. Krishnamurthy, Physical Review B 61, 12739 (1999), arXiv:9903273 [cond-mat] .

[20] T. Maier, M. Jarrell, T. Pruschke, and M. H. Hettler, Reviews of Modern Physics 77, 1027 (2005), arXiv:0404055 [cond-mat] .

[21] G. Kotliar, S. Y. Savrasov, K. Haule, V. S. Oudovenko, O. Parcollet, and C. A. Marianetti, Reviews of Modern Physics 78, 865 (2006).

[22] B. Kyung, D. Sénéchal, and A.-M. S. Tremblay, Physical Review B 80, 205109 (2009), arXiv:0812.1228.

[23] G. Sordi, P. Sémon, K. Haule, and A.-M. S. Tremblay, Physical Review Letters 108, 216401 (2012), arXiv:1201.1283 .

[24] M. Civelli, M. Capone, A. Georges, K. Haule, O. Parcollet, T. D. Stanescu, and G. Kotliar, Physical Review Letters 100, 046402 (2008), arXiv:0704.1486.

[25] M. Ferrero, O. Parcollet, a. Georges, G. Kotliar, and D. N. Basov, Physical Review B 82, 054502 (2010).

[26] E. Gull, O. Parcollet, and A. J. Millis, Physical Review Letters 110, 216405 (2013), arXiv:1207.2490 .

[27] A. Macridin, M. Jarrell, and T. Maier, Physical Review B 70, 2 (2004).
[28] T. a. Maier, M. Jarrell, A. Macridin, and C. Slezak, Physical Review Letters 92, 027005 (2004), arXiv:0211298 [cond-mat] .

[29] T. a. Maier, M. Jarrell, T. C. Schulthess, P. R. C. Kent, and J. B. White, Physical Review Letters 95, 237001 (2005), arXiv:0504529 [cond-mat] .

[30] T. Maier, M. Jarrell, and D. Scalapino, Physical Review Letters 96, 047005 (2006).

[31] E. Gull, M. Ferrero, O. Parcollet, A. Georges, and A. J. Millis, Physical Review B 82, 155101 (2010).

[32] S. X. Yang, H. Fotso, S. Q. Su, D. Galanakis, E. Khatami, J. H. She, J. Moreno, J. Zaanen, and M. Jarrell, Physical Review Letters 106, 047004 (2011), arXiv:1101.6050 .

[33] A. Macridin and M. Jarrell, Physical Review B 78, 241101(R) (2008), arXiv:0806.0815 .

[34] A. Macridin, M. Jarrell, T. Maier, P. R. C. Kent, and E. D'Azevedo, Physical Review Letters 97, 036401 (2006), arXiv:0509166 [cond-mat] .

[35] M. Jarrell, T. Maier, C. Huscroft, and S. Moukouri, Physical Review B 64, 195130 (2001), arXiv:0108140 [cond-mat] .

[36] D. Bergeron, V. Hankevych, B. Kyung, and A.-M. S. Tremblay, Physical Review B 84, 085128 (2011).

[37] B. Kyung, V. Hankevych, A.-M. Daré, and A.-M. Tremblay, Physical Review Letters 93, 147004 (2004).

[38] B. Kyung, S. S. Kancharla, D. Sénéchal, and A.-M. S. Tremblay, Physical Review B 73, 165114 (2006).

[39] S. Okamoto, D. Sénéchal, M. Civelli, and A.M. S. Tremblay, Physical Review B 82, 180511 (2010), arXiv:1008.5118.

[40] G. Sordi, K. Haule, and A.-M. S. Tremblay, Physical Review Letters 104, 226402 (2010).

[41] G. Sordi, P. Sémon, K. Haule, and a.-M. S. Tremblay, Scientific Reports 2, 547 (2012), arXiv:1110.1392 .

[42] M. Civelli, M. Capone, S. S. Kancharla, O. Parcollet, and G. Kotliar, Physical Review Letters 95, 106402 (2005), arXiv:0411696 [cond-mat] .

[43] M. Ferrero, P. S. Cornaglia, L. De Leo, O. Parcollet, G. Kotliar, and A. Georges, Europhysics Letters 85, 57009 (2008), arXiv:0806.4383.

[44] M. Ferrero, P. Cornaglia, L. De Leo, O. Parcollet, G. Kotliar, and A. Georges, Physical Review B 80, 064501 (2009).

[45] E. Gull, O. Parcollet, P. Werner, and A. J. Millis, Physical Review B 80, 245102 (2009), arXiv:0909.1795.

[46] N. S. Vidhyadhiraja, A. Macridin, C. Sen, M. Jarrell, and M. Ma, Physical Review Letters 102, 206407 (2009), arXiv:0909.0498 .

[47] P. Sun and G. Kotliar, Physical Review B 66, 1 (2002).

[48] P. Sun and G. Kotliar, Physical Review Letters 8019, 23 (2004), arXiv:0312303v2 [arXiv:cond-mat] .

[49] S. Biermann, F. Aryasetiawan, and A. Georges, Physical Review Letters 90, 086402 (2003).

[50] T. Ayral, P. Werner, and S. Biermann, Physical Review Letters 109, 226401 (2012).

[51] T. Ayral, S. Biermann, and P. Werner, Physical Review B 87, 125149 (2013).

[52] P. Hansmann, T. Ayral, L. Vaugier, P. Werner, and S. Biermann, Physical Review Letters 110, 166401 (2013).

[53] L. Huang, T. Ayral, S. Biermann, and P. Werner, Physical Review B 90, 195114 (2014), arXiv:1404.7047 .

[54] A. Toschi, A. Katanin, and K. Held, Physical Review B 75, 045118 (2007). 
[55] A. Katanin, A. Toschi, and K. Held, Physical Review B 80, 075104 (2009).

[56] T. Schäfer, F. Geles, D. Rost, G. Rohringer, E. Arrigoni, K. Held, N. Blümer, M. Aichhorn, and A. Toschi, Physical Review B 91, 125109 (2015), arXiv:1405.7250.

[57] A. Valli, T. Schäfer, P. Thunström, G. Rohringer, S. Andergassen, G. Sangiovanni, K. Held, and A. Toschi, arXiv (2014), arXiv:arXiv:1410.4733v1 .

[58] A. N. Rubtsov, M. I. Katsnelson, and A. I. Lichtenstein, Physical Review B 77, 033101 (2008), arXiv:0612196 [cond-mat] .

[59] A. N. Rubtsov, M. I. Katsnelson, and A. I. Lichtenstein, Annals of Physics 00, 17 (2012), arXiv:1105.6158 .

[60] H. Hafermann, E. G. C. P. van Loon, M. I. Katsnelson, A. I. Lichtenstein, and O. Parcollet, Physical Review B 90, 235105 (2014).

[61] C. Taranto, S. Andergassen, J. Bauer, K. Held, A. Katanin, W. Metzner, G. Rohringer, and A. Toschi, Physical Review Letters 112, 196402 (2014).

[62] C. D. Dominicis and P. Martin, Journal of Mathematical Physics 5, 14 (1964).

[63] C. D. Dominicis and P. Martin, Journal of Mathematical Physics 5, 31 (1964).

[64] A. M. Sengupta and A. Georges, Physical Review B 52, 10295 (1995).

[65] H. Kajueter, Interpolating Perturbation Scheme for Correlated Electron Systems, Ph.D. thesis (1996).

[66] Q. Si and J. L. Smith, Physical Review B 77, 3391 (1996), arXiv:9606087 [cond-mat] .

[67] F. Aryasetiawan and S. Biermann, Physical Review Letters 100, 116402 (2008).

[68] A. Georges and G. Kotliar, Physical Review B 45, 6479 (1992).

[69] T. Ayral and O. Parcollet, in preparation .

[70] J. Kuneš, Physical Review B 83, 085102 (2011), arXiv:1010.3809 .

[71] A. N. Rubtsov, V. V. Savkin, and A. I. Lichtenstein, Physical Review B 72, 035122 (2005), arXiv:0411344 [cond-mat] .

[72] P. Werner, A. Comanac, L. de' Medici, M. Troyer, and A. Millis, Physical Review Letters 97, 076405 (2006).

[73] P. Werner and A. Millis, Physical Review Letters 99, 1 (2007).

[74] J. Otsuki, Physical Review B 87, 125102 (2013), arXiv:1211.5935 .

[75] H. Hafermann, Physical Review B 89, 235128 (2014), arXiv:arXiv:1311.5801v1 .

[76] O. Parcollet, M. Ferrero, T. Ayral, H. Hafermann, P. Seth, and I. S. Krivenko, in preparation .

[77] A. Damascelli, Z. Hussain, and Z. Shen, Reviews of Modern Physics 75 (2003).

[78] B. Kyung and G. Kotliar, Physical Review B 73, 205106 (2008), arXiv:0601271v2 [arXiv:cond-mat] . 


\section{Supplementary Materials}

\section{Appendix A: Atomic Vertex}

In the atomic limit (single atomic site), one can compute the three-point vertex exactly by writing its Lehmann representation. One gets the following expression [69]:

$$
\begin{aligned}
\Lambda_{\mathrm{at}}^{\eta=\mathrm{ch} / \mathrm{sp}}\left(i \omega_{n}, i \Omega_{m}\right)= & \frac{1}{1-U^{\eta} \chi_{\mathrm{at}}^{\eta} \delta_{m}}\left(\frac{U^{2} / 4}{i \omega_{n}\left(i \omega_{n}+i \Omega_{m}\right)}+1\right. \\
& \left.+\frac{U \beta\left\langle n_{\sigma}\right\rangle}{2}\left\{1-\frac{U^{2}}{4\left(i \omega_{n}\right)^{2}}\right\}\left\{\tanh \left(\frac{\beta U}{4}\right) \mp 1\right\} \delta_{m}\right)
\end{aligned}
$$

where $\chi_{\mathrm{at}}^{\eta=\mathrm{ch} / \mathrm{sp}} \equiv \frac{\beta}{2} \frac{e^{\mp \beta U / 4}}{\cosh (\beta U / 4)}$ and at half-filling, $\left\langle n_{\sigma}\right\rangle=1 / 2$.

\section{Appendix B: Computation of the three-leg vertex}

$\Lambda_{i m p}$ is computed from the fermionic three-point correlation function through the relation:

$$
\Lambda_{\mathrm{imp}}^{\eta}(i \omega, i \Omega)=\frac{\tilde{\chi}_{\mathrm{imp}, \mathrm{c}}^{\eta}(i \omega, i \Omega)}{G_{\mathrm{imp}}(i \omega) G_{\mathrm{imp}}(i \omega+i \Omega)\left(1-\mathcal{U}^{\eta}(i \Omega) \chi_{\mathrm{imp}, \mathrm{c}}^{\eta}(i \Omega)\right)}
$$

Here, the suffix "c" stands for "connected", namely:

$$
\tilde{\chi}_{\mathrm{imp}, \mathrm{c}}^{\eta}(i \omega, i \Omega) \equiv \tilde{\chi}_{\mathrm{imp}}^{\eta}(i \omega, i \Omega)-\beta G_{\mathrm{imp}}(i \omega)\left\langle n_{\mathrm{imp}}^{\eta}\right\rangle \delta_{i \Omega}
$$

while $\tilde{\chi}_{\text {imp }}^{\eta}(i \omega, i \Omega)$ is defined as $\tilde{\chi}_{\text {imp }}^{\mathrm{ch} / \mathrm{sp}}(i \omega, i \Omega)=\tilde{\chi}_{\mathrm{imp}}^{\uparrow \uparrow}(i \omega, i \Omega) \pm \tilde{\chi}_{\mathrm{imp}}^{\uparrow \downarrow}(i \omega, i \Omega)$, with:

$$
\tilde{\chi}_{\mathrm{imp}}^{\sigma \sigma^{\prime}}(i \omega, i \Omega) \equiv \iint_{0}^{\beta} d \tau d \tau^{\prime} e^{i \omega \tau+i \Omega \tau^{\prime}}\left\langle T c_{\sigma}(\tau) c_{\sigma}^{\dagger}(0) n_{\sigma^{\prime}}\left(\tau^{\prime}\right)\right\rangle
$$

$\chi_{\mathrm{imp}}^{\eta}(i \Omega)$ is the Fourier transform of $\chi_{\mathrm{imp}}^{\eta}(\tau) \equiv\left\langle T n^{\eta}(\tau) n^{\eta}(0)\right\rangle_{\mathrm{c}}$.

\section{Appendix C: Inverse spin susceptibility: Temperature evolution}

The inverse static AF spin susceptibility $\left(\chi^{\mathrm{sp}}(\mathbf{Q}, i \Omega=0)^{-1}\right.$, obtained from $W^{\mathrm{sp}}(\mathbf{q}, i \Omega)$ by Eq. (9)) decreases linearly with temperature, as shown in Fig. A.1. This allows to determine $T_{\mathrm{AF}}$ by extrapolation. The resulting phase diagram is shown in Fig. 2.
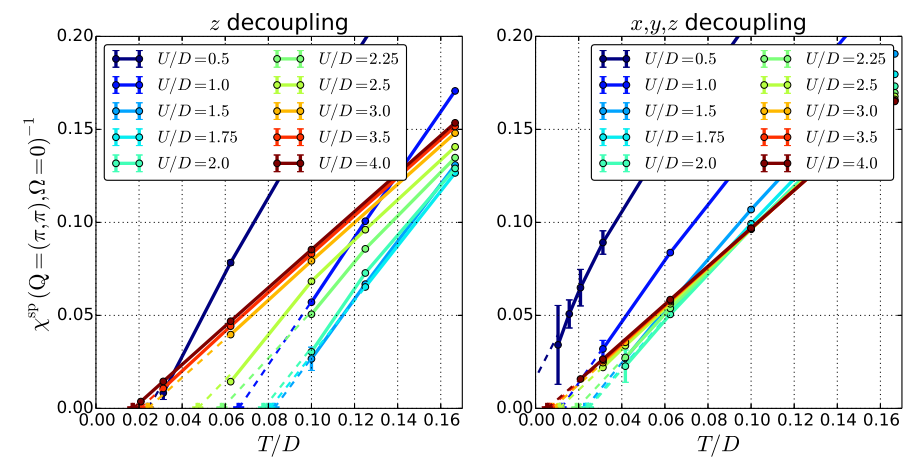

Figure A.1: (color online) Evolution of the inverse susceptibility with temperature (half-filling, $t^{\prime}=0$ ). The circles denote TRILEX computations, while the crosses on the $x$-axis are the extrapolated points. The dashed portion is the extrapolated line. Left: $z$-decoupling. Right: $x y z$-decoupling. 